Volker Bruns*, Daniela Franz, Petr Kuritcyn, Veit Wiesmann, Magnus Rathke, Thomas Wittenberg, Alexandra Wießner, Laura Kursawe, Annette Moter, Judith Kikhney*, Christian Münzenmayer*

\title{
Towards computer aided diagnosis of infective endocarditis in whole-slide images of heart valve tissue using FISH
}

\begin{abstract}
Infective endocarditis (IE) is an infection of the endocardium, and the heart valves associated with high morbidity and mortality. Fluorescence in situ Hybridization (FISH) is a molecular imaging technique used for diagnosis of IE based on histological heart valve tissue sections. FISH allows detection and identification of microorganisms and gives information about their quantity and spatial distribution. This information is important to guide appropriate antibiotic treatment. However, as manual FISH image analysis is time- and costexpensive, an automated image analysis pipeline (consisting of tissue segmentation, bacteria detection, and spot detection modules) is proposed to assist locating potential regions with microorganisms.

The proposed approach was evaluated in a study, where five observers manually assessed a set of 171 fields-of-view (FoVs) captured in 400-fold magnification from 10 randomly chosen WSI for the presence of microorganisms, morphologically detected by the nucleic acid stain DAPI. The task of the observers was to mark the presented image using a 2-class score ('positive/questionable' or 'negative'). The human assessment was compared to the results suggested by the algorithm. The proposed algorithm locates and ranks potential regions with microorganisms in heart valve sections so that experts can validate them in higher power FoVs for the presence of bacteria and identify their species. The automated system for preselecting and recommending adequate FoVs is thus a starting point to support experts and save human resources. It is now ready to be further developed for the detection of bacteria by FISH.
\end{abstract}

Keywords: FISH, image analysis, manual assessment

https://doi.org/10.1515/cdbme-2021-2119

\footnotetext{
*Corresponding author: V Bruns: Fraunhofer Institute for Integrated Circuits IIS, Am Wolfsmantel 33, 91058 Erlangen, Germany, e-mail: volker.bruns@fraunhofer.de. D Franz, P Kuritcyn, C Münzenmayer, M Rathke, $\mathbf{V}$ Wiesmann, $\mathbf{T}$ Wittenberg: Fraunhofer IIS, Erlangen; J Kikhney, L Kursawe, A Wießner, A Moter: Charité-University Medicine Berlin, Germany, MoKi Analytics $\mathrm{GmbH}$, Hindenburgdamm 30, 12203 Berlin, Germany

- Both senior authors have contributed equally
}

\section{Introduction}

In infective endocarditis (IE), microorganisms grow in the tissue of the endocardium and the heart valves leading to high morbidity and mortality [1]. Timely diagnosis and identification of the causative pathogen followed by selected antibiotic therapy is crucial for the patient's outcome. The standard diagnostic tests include cultures from blood and heart valve samples. However, these tests frequently fail to grow the pathogen, in particular when the patient already was treated with antibiotics. We apply fluorescence in situ hybridization (FISH) in combination with PCR and sequencing (FISHseq) to detect and to identify microorganisms in heart valve tissue, even in culture-negative cases [2]. This information is important to select appropriate antibiotic treatment.

However, the detection of bacteria in heart valve tissue samples using FISH is a tedious process that is time consuming and costly. Due to the small size of the bacteria in the range of $5 \mu \mathrm{m}$ down to $0.5 \mu \mathrm{m}$ (which is $\sim 10 \%$ of the size of eukaryotic cells), visual inspection using a microscope requires a strong magnification. With a 400 -fold magnification, bacteria are barely visible as small inter-cellular spots in the DAPI signal, see Figure 1. For identification and quantification of the bacteria, a 1,000-fold magnification is required and comprises the combination of DAPI with bacteria-specific FISH-probes as well as a nonsense FISH probe and an empty Fluorescence channel to exclude unspecific probe binding or autofluorescence [3]. While a 'positive' diagnosis can stop as soon as one field-ofview (FoV) showing identifiable microorganisms is found, a 'negative' or 'questionable' diagnosis requires the entire valve tissue sample to be eyeballed while switching back and forth between multiple fluorescence channels. Even for experienced users, this process may takes at least 30 minutes per sample, which amounts to one hour in total for a first and second look of two experts.

Thus, this research addresses the question how automated image analysis can assist locating potential regions with microbial infection, and hence aid the diagnosis of IE using FISH imaging. In a first step, we developed an algorithm to identify regions of interest by detecting bacteria with the DAPI stain only. 


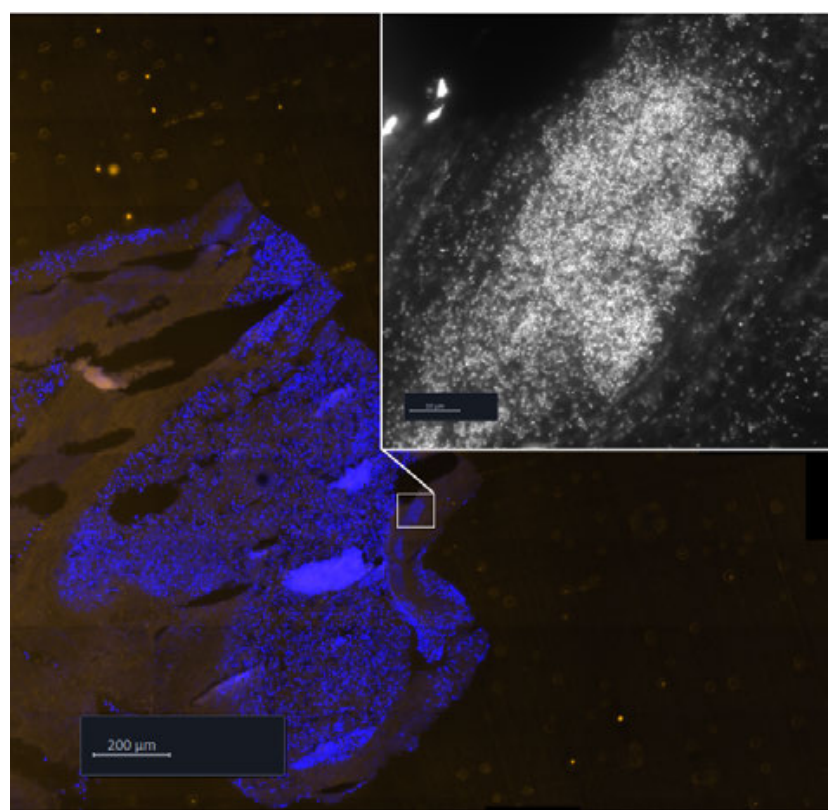

Figure 1. Large: contrast-enhanced overview megapixel image (400-fold magnification, $0.114 \mathrm{um} / \mathrm{pixel}$ ), blue: DAPI stains DNA. Small: Detail (400-fold magnification), DAPI only, showing microorganisms.

\section{Related Work}

FISH imaging has in the past been proposed as a strong diagnostic tool for the detection of microorganisms in clinical microbiology, including blood [4], wounds [5], or medical devices [6]. FISH for identifying microorganisms in infected heart valve tissue has recently been described by Eichinger et al. [7]. Other works in the same context focus on fastidious or unculturable bacterial species in IE, e.g., Coxiella burnetii. With respect to automated image analysis, only few works are available in this context. Selinummi et al. [8] described a thresholding approach segmenting bacteria in FISH images; however, the examined images were smears without tissue and therefore had no considerable background signal. For the task of mitochondria segmentation Peng et al. [9] proposed to use an adaptive local thresholding. Held et al. [10] evaluated maximum-intensity-linking and watershed segmentation algorithms on various fluorescent microscope images and proposed improvement for both approaches. They conclude that the watershed approach is superior for a variety of fluorescence images. They also proposed a thresholding algorithm that is trained based on manual annotations [8].

\section{Data}

For developing and evaluating an image analysis algorithm for automated detection of microorganisms in tissue sections, a data collection has been compiled based on 43 digitized whole-slide images (WSIs) of tissue samples from patients from the Charite that underwent surgical heart valve replacement due to suspected IE. The set comprises both native and prosthetic heart valves. All samples were analysed by FISHseq. Epifluorescence evaluation of FISH results was carried out using four narrow band filter sets for DAPI: $353 \mathrm{~nm} / 465 \mathrm{~nm}$; FITC: $495 \mathrm{~nm} / 519 \mathrm{~nm}$; Cy3: $548 \mathrm{~nm} / 561 \mathrm{~nm}$; and Cy5: 650 $\mathrm{nm} / 673 \mathrm{~nm}$. All tissue samples were digitized with a Zeiss AxioImager.Z2 whole-slide-scanner. For each sample, an overview scan with a 400-fold magnification was created (s. Fig. 1) and up to ten non-overlapping 1,000-fold FoVs at locations where bacteria are present. A single 400-fold whole-slide had a size of $\sim 60.000^{2}$ pixels.

\section{Methods}

Figure 1 shows on the left side an overview scan of the FISH analysis of a heart valve tissue section captured with a 40 -fold objective. On the right side, an enlarged region of interest (ROI) in the DAPI channel from the same image is depicted containing microorganisms. Taking only the DAPI channel into account, the bacteria to be automatically detected appear as small bright spots and are located within the tissue. Still, specific care needs to be taken to reject small DAPI spots inside or closely adjacent to cell nuclei, which may be related to an uneven distribution of DNA in the nuclei and may be mistaken as bacteria. Furthermore, bacteria can be present either within the heart valve tissue - identified by its auto-fluorescence (green channel) - or located on the heart valve. The image analysis pipeline compromises the following steps:

\subsection{Tissue Segmentation}

The first step is to detect the presence of tissue in a whole slide image and outline its contours. This task of tissue segmentation is solved using adaptive binary thresholding in the autofluorescence (green) channel, as its histogram depicts three distinct modes, related to the background (mode 1 and 2) and the autofluorescence of the tissue (mode 3). An automatically detected threshold between the second and third mode yields a robust detection and masking of the tissue. The borders of this mask can be traced to detect the outer tissue contours and the inner defects (e.g., holes in the tissue).

\subsection{Bacteria Detection}

The detection of bacteria consists of multiple steps (see Fig. 2). The DAPI image is initially processed separately in two arms: one arm detects single spots (presumable bacteria), 
while the other arm detects cells and bright areas, which are masked and discarded. In the spot-detection arm, the DAPI image is initially processed with a feature enhancing Difference-of-Gaussian (DoG) filter by subtracting two different Gaussian-blurred versions of the image from each other $\left(\sigma_{1}=1.0\right.$, $\sigma_{2}=3.0$ ). The resulting image is binarized using a $k$-means clustering with $k=3$, where the brightest cluster is selected to be the foreground. The remaining foreground is then segmented into individual objects, each representing a possible bacterium. Nevertheless, these objects are evaluated by their area $A_{i}$ and average brightness $B_{i}$, where only candidates with $A_{i} \in[5$ -50] pixels (equivalent to a range of $[0.06-0.6] \mu \mathrm{m}^{2}$ ) and $B_{i}$, $>3$ are kept (Fig. 2, top row).

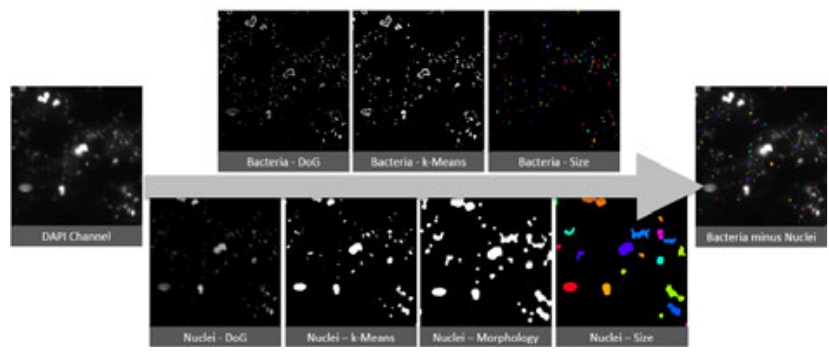

Figure 2: Bacteria detection algorithm. See explanation above.

However, the thus remaining set of objects may still include spots not corresponding to bacteria. In particular, spots inside host cell nuclei need to be removed as these are more likely related to an inhomogeneous DNA distribution inside the nucleus. In addition, the DAPI channel may exhibit a background signal due to the long exposure time that is required for visualization of bacteria. Thus, in these areas with inhomogeneous background false positive signals may be mistaken as bacteria and need to be rejected. Therefore, to detect possible nuclei in the DAPI images (Fig. 2, bottom), again a DoG filter $\left(\sigma_{1}=1.0\right.$, $\left.\sigma_{2}=30.0\right)$ is applied followed by a k-means binarization $(k=3)$. A morphological closing with Chamfer-distance of 15 px is used to close small holes in the nuclei. The detected nuclei objects morphologically delated ( $r=5 \mathrm{px}$ ) to include also fainter nuclei borders. Finally, smaller regions with an area $A_{i}$ $<300 \mathrm{px}$ (equiv. to $34.2 \mu \mathrm{m}^{2}$ ) are removed as these are unlikely to be cell nuclei.

\subsection{Detection of Regions of Interest}

The described detection of bacteria is applied successively to all FoVs in all WSIs where an intersection exists to the previously detected foreground mask (s. Sec. 4.1). Next, all detected spots are clustered into non-overlapping rectangular clusters with a size of a single 1,000-fold (high power) field with optical resolution of $125 \times 100 \mu \mathrm{m}^{2}$. With this approach, it is possible to provide and visualize the top $N$ FoVs based on the number of contained spots to a human expert, who is then responsible for the final evaluation. To ensure that regions of interest from all parts of the tissue sample are represented, an additional constrain is introduced, by overlaying a coarsely grained virtual grid ( $6 \times 3$ cells) onto the WSI and listing the top $N$ FoVs as a virtual grid.

\section{Experiments and Results}

\subsection{Inter-observer study}

For the evaluation of the proposed strategy of automatic preselection of adequate FoVs in FISH images, a study was carried out to analyse the degree of concordance between the ROIs proposed by image analysis and their classification by the human observers. Thus, five observers experienced in FISH data assessment were asked to analyse FISH images from heart valves clinically suspected for IE. The test data set (disjunct from data used for algorithm development) consisted of 10 WSIs (400x magnification) with 171 FoVs containing 'positive / questionable', or 'negative' areas (pre-analysed by FISH with respect to IE) from native and prosthetic heart valves and different bacterial species (staphylococci, streptococ$c i$, enterococci, others). Participants were asked to view the WSIs (using the Zeiss Zen slide viewer) together with overlay files related to the top 5 FoVs from the virtual grid (see Sec. 4.3 ) indicated by the number of detected spots. The overlayed annotations had unique IDs and were color-coded according to the number of automatically detected spots in the FoV. The users categorized every FoV as 'positive' / 'questionable' (as ROIs be evaluated in $1,000 \mathrm{x}$ magnification with all four fluorescence channels) versus 'negative'.

\subsection{Results}

A total of 855 answers ( 5 observers, 171 FoVs) were provided, see Fig. 3. The distribution amongst the three classes ("positive / questionable' / 'negative') was 43.3\%, 31.9\%, 24.8\%, implicating that $25 \%$ of the FoVs did not contain any microorganisms despite automatically detected spots. The sum of 'positive' / 'questionable' answers for observers B-E is almost constant, while observer A significantly found more images to be 'negative'. Fig. 4 provides a breakdown of automatically assessed FoVs into six classes based on the number $b$ of automatically detected bacteria: $c_{1}: b>2,500 ; c_{2}: 1000<b \leq 2,500$; $\mathrm{c}_{3}: 500<b \leq 1,000 ; \mathrm{c}_{4}: 100<b \leq 500 ; \mathrm{c}_{5}: 10<b \leq 100 ; \mathrm{c}_{6}: b \leq$ $10)$. For the classes $c_{1}$ to $c_{3}$, the ratio of 'positive' and 'questionable' votes is over $90 \%$, indicating the proposed algorithm is quite good. The answers of the observers in the algorithm 
categories $\mathrm{c}_{4}$ and $\mathrm{c}_{5}$ is distributed, whereas the last category $\mathrm{c}_{6}$ is rather equivalent with the observer's answers.
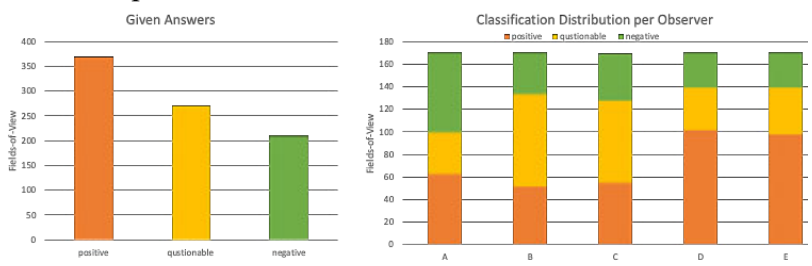

Figure 3: left: answers per class; right: answers per observers.

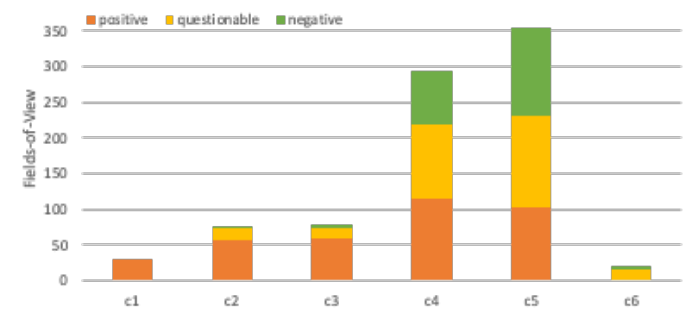

Figure 4: automatically assessed FoVs (in 6 classes, ranging from $\mathrm{c}_{1}>2,500$ bacteria to $\mathrm{c}_{6}$ : $<10$ bacteria) compared to human observers (3 classes).

\section{Discussion and Conclusion}

The described work focused on image analysis methods for the computer-assisted diagnosis of infective endocarditis based on FISH data. The proposed algorithm locates and ranks areas of potential microbial infections in 400-fold magnification WSIs in order to present them in higher power FOVs (in 1,000-fold magnification) to qualified experts to validate the presence of bacteria and identifying their species. Such an automated and objective system for preselecting and recommending adequate FoVs can save expensive human resources needed to browse through the complete WSI data at multiple magnifications and search for regions of interest. Overall, the proposed algorithm showed a good performance when high amounts of bacteria were present. Nevertheless, for practical application, still too many images were automatically proposed containing possible bacteria. This must be addressed in the next steps. Also, a third of the human voters classified the FOVs as 'questionable', indicating that the used information from 400 -fold magnification is not sufficient. Therefore, future work should include the automated analysis of images with 1,000-fold magnification as are used for human inspection.
Acknowledgement: This work was carried out as part of the iSOLID project (13N 14396) funded by the German Ministry for Education \& Research (BMBF) under 'Photonics in Life Sciences' program

\section{References}

[1] Habib G, Lancellotti P, Antunes MJ et al.: 2015 ESC Guidelines for management of infective endocarditis: Europ. Heart J. 36(44) 2015, pp. 3075-3128

[2] Mallmann C, Siemoneit S, Schmiedel D, et al.: Fluorescence in situ hybridization to improve the diagnosis of endocarditis: a pilot study. Clin Microbiol Infect 2010; 16: 767-77.

[3] Kikhney J, Moter A: Quality control in diagnostic fluorescence in- situ hybridization in microbiology methods. Mol Biol. 2021; 2246: 301-316.

[4] Kempf VA, Trebesius K, Autenrieth IB. Fluorescent In situ hybridization allows rapid identification of microorganisms in blood cultures. J Clin Microbbiol. 38(2):830-838, 2000.

[5] Malic S, Hill KE, Hayes A, Percival SL: Detection and identification of specific bacteria in wound biofilms using peptide nucleic acid fluorescent in situ hybridization. Microbiology 2009, 155 (Pt 8): 2603-2611.

[6] Xu Y, Larsen LH, Lorenzen J, Hall-Stoodley L: Microbiological diagnosis of device-related biofilm infections. APMIS 125: 289-303

[7] Eichinger S, Kikhney J, Moter A, et al.: Fluorescence in situ hybridization for identification and visualization of microorganisms in infected heart valve tissue. Inter.Cardiovasc \& Thoracic Surg 2019.

[8] Selinummi J, Seppälä J, Yli-Harja O, Puhakka JA. Software for quantification of labeled bacteria from digital microscope images by automated image analysis. BioTechniques 2005, 39:6, 859-863.

[9] Peng JY. Adaptive local thresholding for fluorescence cell micrographs Technical Rep. No. TR-IIS-09-008 (2009).

[10] Held C, Palmisano R, Häberle L, Hensel M, Wittenberg T. Comparison of parameter-adapted segmentation methods for fluorescence micrographs. Cytom. A 2011, 79, 933-45. 